\title{
Temporomandibular joint arthrocentesis: an evidence-based review
}

\author{
M. Franklin Dolwick ${ }^{1}$, Charles G. Widmer ${ }^{2}$ \\ ${ }^{1}$ Department of Oral and Maxillofacial Surgery, College of Dentistry, ${ }^{2}$ Division of Facial Pain, Department of Orthodontics, College of Dentistry, \\ University of Florida, Gainesville, FL, USA \\ Contributions: (I) Conception and design: All authors; (II) Administrative support: None; (III) Provision of study materials or patients: None; (IV) \\ Collection and assembly of data: All authors; (V) Data analysis and interpretation: All authors; (VI) Manuscript writing: All authors; (VII) Final \\ approval of manuscript: All authors. \\ Correspondence to: Charles G. Widmer, DDS, MS. Parker E. Mahan Facial Pain Endowed Professor, Department of Orthodontics, College of \\ Dentistry, University of Florida, 1395 Center Drive, Gainesville, FL 32610-0444, USA. Email: widmer@dental.ufl.edu.
}

Background and Objective: Temporomandibular joint (TMJ) intracapsular pain can sometimes persist after pursuing reversible techniques. TMJ pain reduction may require a more focused treatment approach such as arthrocentesis to remove intracapsular inflammatory mediators. Evidence of efficacy for treating TMJ pain and mandibular mobility using TMJ arthrocentesis is available from a small number of high-quality evidence randomized controlled trials (RCTs). However, there still remains a lack of high-quality evidence to test the efficacy of various supplements that have been proposed to further enhance the outcomes after arthrocentesis. The purpose of this review was to examine published RCTs to determine the efficacy of TMJ arthrocentesis alone and with supplementation by steroids, hyaluronic acid and platelet-rich plasma growth factors.

Methods: A literature search in MEDLINE, EMBASE and CENTRAL databases was completed on July 1, 2020 to identify randomized clinical trials that studied the efficacy of temporomandibular joint arthrocentesis with or without supplements on primary outcome measures of pain and mandibular mobility. Studies were also identified from reference lists of review articles or systematic reviews. The literature search spanned the years 1989-2020 and only articles written in English were evaluated. Search terms included "temporomandibular joint" OR “TMJ” AND "arthrocentesis".

Key Content and Findings: Thirty-one RCTs were identified from the literature with most of the studies having low to medium quality evidence. Most of the RCTs suffered from a lack of controlling experimental bias, lack of inclusion and exclusion criteria, lack of pain source validation, lack of stratification by sex and inclusion of bilateral TMJ arthrocentesis procedures that can confound the interpretation of the results of the procedure. Some RCTs were single or double blind studies, but the majority did not use a blinded experimental design. Low to medium quality evidence RCTs that focused on a comparison of TMJ arthrocentesis alone to post arthrocentesis supplementation found no differences in resting pain intensity or mandibular mobility. However, one high-quality evidence RCT that evaluated steroid supplementation (triamcinolone acetonide) found a significant improvement and normalization of chewing pain and the normalization of mandibular mobility at 12 weeks post-arthrocentesis compared to the control group. Hyaluronic acid has been evaluated by medium quality evidence RCTs and these studies did not find differences between supplementation and control groups for pain or mandibular mobility. Finally, plateletrich plasma has been used as a supplement and one study with medium quality evidence found significant differences between groups for pain, mandibular mobility and chewing efficiency.

Conclusions: The paucity of high-quality evidence RCTs has not allowed a consensus for the efficacy of TMJ post-arthrocentesis supplements and further studies are required to guide the practicing clinician on their use.

Keywords: Temporomandibular joint (TMJ); pain; arthrocentesis; efficacy; randomized clinical trial

Received: 06 August 2020; Accepted: 10 October 2020; Published: 29 October 2020.

doi: 10.21037/fomm-20-51

View this article at: http://dx.doi.org/10.21037/fomm-20-51 


\section{Introduction}

Temporomandibular joint (TMJ) arthrocentesis has gained popularity as a minimally invasive technique to effectively manage intra-capsular TMJ pain and mandibular mobility restriction secondary to TMJ internal derangements such as an anteriorly displaced disk without reduction (or closed lock) (1-8). More recent studies have duplicated the results of earlier studies (9-33). The original article by Nitzan et al. (1) reported placement of two needles into the TMJ superior joint space under local anesthesia and lavaging the joint with lactated Ringer's solution to normalize translation of the disk and condyle in patients with a closed lock condition and to reduce pain. All 17 patients reported a sudden limitation in mandibular opening prior to TMJ arthrocentesis that was consistent with either an acute anterior displaced without reduction or an articular disk adhered to the fossa due to a vacuum effect caused presumably from clenching. Both of these conditions would respond favorably to TMJ arthrocentesis. In fact, all patients had an improved mandibular mobility measured at four months or later post-arthrocentesis and 16/17 (94\%) had substantial pain reduction. A few years later, the addition of various medications to supplement the lavage technique was reported with anecdotal outcomes of success. More recently, randomized controlled trials (RCTs) have been conducted to determine the efficacy of the TMJ arthrocentesis procedure for a variety of TMJ arthralgia conditions and most have reported a significant decrease of resting pain intensity and limited mandibular mobility over time but no significant difference between the TMJ arthrocentesis procedure and the additional supplementation of a medication. However, there are confounding factors in these studies that complicate the interpretation of the results.

The purpose of this review was to evaluate and discuss the strengths and weaknesses of these evidence-based studies and to provide recommendations for clinical management of intra-articular pain using TMJ arthrocentesis. Medication supplements after TMJ arthrocentesis such as a steroid or hyaluronic acid or autologous supplements such as platelet rich plasma were also reviewed to determine their additional efficacy.

\section{Methods}

The search for relevant literature was completed on July 1, 2020 in MEDLINE, EMBASE and CENTRAL (Cochrane Central Registry of Controlled Trials) databases.
Relevant studies were also identified from reference lists of review articles or systematic reviews of TMJ arthrocentesis. Articles were considered for review if they were published in English between 1989-2020. The search terms included any studies that had "temporomandibular joint" OR "TMJ" AND "arthrocentesis". Identified studies from this search strategy were further examined for the word "randomized" to separate clinical reports or retrospective studies from randomized clinical trials. The article designation as an RCT was independently verified by both authors (agreement was unanimous). Each article was evaluated for the randomization process, blinding procedures, patient description (sex, TMJ diagnosis), inclusion/exclusion criteria, type of anesthesia (local, IV sedation with local), unilateral or bilateral TMJ pain, placebo group, passive lavage or active distension of TMJ during arthrocentesis, appropriate statistics applied, sample size calculation, follow-up duration, primary and secondary outcome measures, pain assessment measures, supplemental medications after TMJ arthrocentesis and effectiveness of the procedure on pain and mandibular mobility.

\section{Identifying the appropriate patient to test TMJ arthrocentesis efficacy}

TMJ arthrocentesis is a technique that effectively manages intracapsular pain conditions such as inflammation (retrodiscitis, osteoarthritis) and displaced disk mobility conditions within the TMJ. The lavaging action decreases inflammatory mediators and cytokines that are responsible for pain $(34,35)$ and lubricates the superior joint space to allow the disk to move without restriction. Also, distension of the condyle from the eminence during the procedure can effectively relieve small adhesions that may have formed also improving mandibular mobility. This procedure is usually considered after patients have been initially treated using medications such as non-steroidal anti-inflammatory drugs (NSAIDs) or muscle relaxants, physical modalities, behavioral management and an occlusal appliance to treat both the joint and muscle pain conditions. To be considered a candidate for a successful TMJ arthrocentesis procedure, patients should subsequently present with predominant TMJ pain and minimal muscle pain after the initial treatment regimen. During our examination of published RCTs testing the efficacy of TMJ arthrocentesis, it was important to determine if the patient sample was identified using appropriate inclusion and exclusion criteria to exclude predominant pain from other sources such as muscle. It 
was also important that well-defined physical examination techniques such as the Research Diagnostic Criteria for Temporomandibular Disorders (RDC/TMD) (36) or other validated techniques were used to identify the presence or absence of signs and symptoms that defined the patient sample. In our review of 31 published RCTs, some studies had no inclusion or exclusion criteria $(2,14,33)$ or used a diagnosis of disk displacement with or without reduction as the inclusion criteria without reporting the exclusion of other pain sources such as masticatory muscle myalgia (3-5,7-11,15,17,18,20-22,24,26-32). Only a few RCTs confirmed the source of pain was from TMJ innervated structures by either the use of a local anesthetic to anesthetize the auriculotemporal nerve and eliminating the TMJ pain (6) or by selectively loading the TMJ by contralateral biting and duplicating the intracapsular source of pain within the joint (19).

Based on our experience, there seems to be a need to distinguish between capsular pain and intracapsular pain associated with the TMJ. Pain relief after TMJ arthrocentesis is more reliably achieved when the pain is from an intracapsular source such as the retrodiscal tissues or from bone to bone contact in inflammatory conditions such as osteoarthritis. Although there are no studies currently available to document this differential success, it has been our experience that capsular pain identified by palpable tenderness lateral to the TMJ is not predictably eliminated by lavage of the joint using $100 \mathrm{~mL}$ of lactated Ringers solution and supplementation of a steroid (triamcinolone acetonide). Therefore, we used a positive response to TMJ loading as a diagnostic criterion for selection of patients for management of articular pain using TMJ arthrocentesis (19).

Another important inclusion criterion that was used in some RCTs of TMJ arthrocentesis was unilateral TMJ pain $(3,14,16,18,19,22,23,25,29,31,32)$. Since all of the studies used pain as one of the primary or secondary outcome measures, the inclusion of bilateral TMJ pain would have been problematic if the treatment was only applied to the joint with the highest level of pain and residual pain continued in the opposite joint or if both TMJs were treated and only one was successfully managed. Mandibular mobility and pain assessments could be affected by TMJ pain that was unresolved after bilateral TMJ arthrocentesis and this residual pain would confound the interpretation of the results of the study. Review of the 31 RCTs for TMJ arthrocentesis efficacy found that only 9 studies (29\%) had inclusion criteria that would specifically identify unilateral TMJ pain patients for participation in the study.

\section{RCTs evaluating TMJ arthrocentesis efficacy and research design issues}

RCTs are considered the best evidence compared to most other types of studies due to the ability to compare treatment outcomes, minimize bias by the use of blinding, and evaluate a homogeneous group of patients that are defined by specific inclusion and exclusion criteria (see above) to minimize selection bias. In review of the RCTs for TMJ arthrocentesis, the randomization process used to assign a patient to a specific treatment group was accomplished by a computer-generated random number list (seven studies), a randomized table/list (three studies) or a sealed envelope methodology (four studies). However, in a majority of the 31 RCTs reviewed, no description was provided regarding the methodology of randomization. One study used a set sequence rather than random assignment and classified their study as an RCT (7) and this misinterpretation of random assignment suggests that a description of the methodology is needed to determine if the study has been properly conducted.

Many of the early RCTs lacked the appropriate research design to minimize bias. Bias can exist in many different forms and can affect the ability to accurately test for differences in treatment outcomes [for review, see table 3 in Dolwick and Widmer (37)]. Bias can be observed in studies where the investigator and patient know the type of treatment or medication that is being used and have a preconceived expectation for the outcome such as comparing TMJ arthrocentesis alone to TMJ arthrocentesis with a supplement such as a steroid or hyaluronic acid (HA). A double-blind study involves blinding the patient and the examiner to minimize bias towards an outcome that is anticipated by the patient and/or examiner to be beneficial. A double-blind study is the gold standard of research design in an RCT. Less than half of the TMJ arthrocentesis RCT studies were blinded (13/31 or $42 \%)$ and less than a quarter of the studies were double-blind studies ( $7 / 31$ or $23 \%$ ). The use of a placebo supplement compared to a medication supplement after arthrocentesis was an additional method to control for bias and was used in 6/31 (or 19\%) of the RCT studies and all were double-blinded. These data support the observation that there was a large variation in the quality of the research design in these RCT studies and most did not adequately evaluate the efficacy of different techniques and their success due to bias.

Another methodological consideration that must be given to studies of pain in the orofacial region is the 


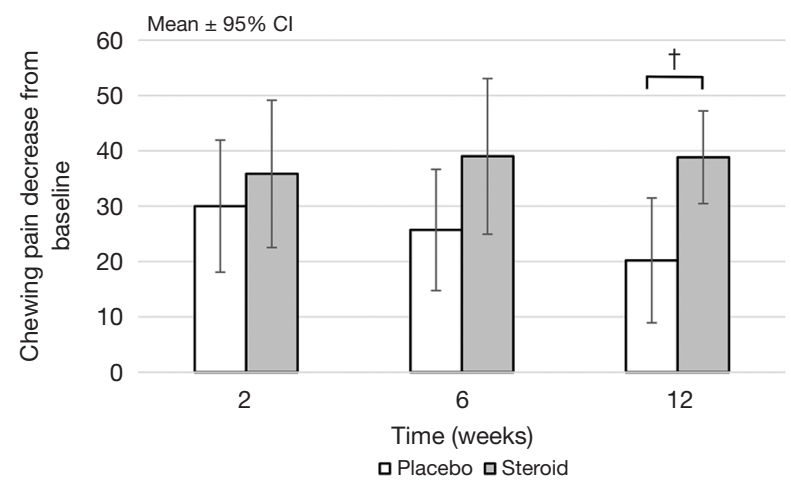

Figure 1 Bars representing the means and 95\% confidence intervals for the magnitude of Visual Analog Scale (VAS) change in chewing pain for placebo and steroid patient groups at various timepoints (2, 6 and 12 weeks post-TMJ arthrocentesis) and calculated from previously published data (19). Note that the steroid group had a relatively constant reduction in chewing pain over the 12 weeks and was always greater than the placebo pain reduction. The placebo group had a reduction in pain but the reduction was progressively less over the 12 weeks. A statistically significant difference $(\mathrm{P}=0.013)$ was found between the placebo and steroid group mean chewing pain decrease at the $12^{\text {th }}$ week $(\dagger)$.

presence of sex differences. It has been known for a long time that females have a higher prevalence of masticatory musculoskeletal pain compared to males (38) and females respond more robustly to experimental pain conditions such as standardized clenching and the resulting post-exercise muscle soreness (39). Therefore, results of therapeutic studies for orofacial pain should ideally be reported for each sex rather than combining both male and females as a group. Reporting of outcomes by sex was only conducted in $13 \%$ of the RCT studies (4/31) that were reviewed. Therefore, over $85 \%$ of the outcomes reported in RCT studies of TMJ arthrocentesis were not from a homogeneous group of patients and may have led to a higher variability of outcome measures within the group. These multiple methodological issues that have been identified may partially explain the lack of statistical significance between experimental groups that have been reported in many studies. The methodological disparities also limit the ability to evaluate multiple studies using meta-analysis.

\section{Studies of TMJ arthrocentesis efficacy without and with medication supplements}

It has been well-established in RCTs that TMJ arthrocentesis without the addition of medications or growth factors can be effective to reduce pain and improve mandibular mobility over time $(2,3,8,9,11-14,16,17,19$ $22,24,26,27,29,32)$. However, the effectiveness of additional supplements infused into the superior joint space of the TMJ has not been clearly established. There are three supplements that have been evaluated by at least two RCTs to determine the efficacy of these medications or growth factors: steroids, HA and platelet-rich plasma (PRP).

\section{Steroid supplementation to reduce intracapsular inflammation}

Steroid injections into the TMJ have been used for many years to treat inflammatory conditions such as various arthritides and arthralgia resulting from disk displacement with or without reduction. So, it was logical to consider injecting an intra-articular steroid after a TMJ arthrocentesis procedure to further reduce the inflammation and extend the reduction or elimination of pain. Steroids such as dexamethasone $(6,8)$, triamcinolone acetonide $(7,19)$, methylprednisolone acetate $(9,23)$ and betamethasone (13) have been evaluated in TMJ arthrocentesis RCT studies. None of the seven studies found statistical differences for primary outcome variables of resting pain intensity and maximum interincisal opening between the steroid group and the control group. Only one of seven RCT studies found statistically significant differences between secondary outcome measures. The steroid group had a reduction of chewing pain at 6 and 12 weeks, an increase in the number of patients with a greater than $50 \%$ reduction of pain at 12 weeks as well as an improvement of mandibular mobility (maximum mandibular opening $>38 \mathrm{~mm}$ without pain) at 12 weeks in patients with osteoarthritis or disk derangements (19). One explanation for the ability to detect differences between steroid and control groups compared to other RCT studies was the minimization of experimental bias, the inclusion of only females with unilateral TMJ pain, welldefined inclusion and exclusion criteria and the validation of TMJ intra-articular pain. Also, functional pain such as chewing pain rather than resting pain intensity is a better outcome measure to evaluate changes after medication supplementation (Figure 1) but was not used in most RCT studies of TMJ arthrocentesis. Normalization of chewing pain and mandibular mobility at 12 weeks was found to be significantly better for the steroid group and can be valuable outcome measures of efficacy of medication 
supplementation.

\section{HA supplementation after TMF arthrocentesis to improve TMF disk mobility and reduce inflammation}

In TMJ osteoarthritis patients, HA supplementation was not found to significantly decrease pain or increase mandibular mobility over TMJ arthrocentesis alone in two of three RCT studies $(7,17)$. The one study where HA supplementation did improve pain and mandibular mobility had five consecutive, weekly TMJ arthrocentesis procedures with HA supplemented after each procedure (33). However, this study had a majority of male patients compared to other studies where the sample was predominantly female, had a small sample size (10 patients in each group), was not a blinded study and the statistical analyses that were used were not described.

HA supplementation after TMJ arthrocentesis has also been tested in patients with displaced disks with or without reduction. Out of eight RCT studies, two studies reported a significant decrease of resting pain intensity compared to TMJ arthrocentesis alone $(14,21)$, one study found a significant increase in maximum interincisal opening (22) and one study found both a decrease in pain intensity and an increase in maximum opening (2). All four of these studies did not have a blinded examiner to conduct the post-arthrocentesis measures and the lack of experimenter blinding may have allowed a bias. Also, 2 of the 3 studies that found a significant decrease of pain intensity had a majority of males in the treatment groups and the predominance of male subjects could have influenced the outcome measure of pain $(14,21)$ and emphasizes the importance of stratification of the outcome measures by sex. The other four RCT studies did not find significant differences in resting pain intensity or maximum interincisal opening and all four studies used blinded examiners who did not know the treatment group of the patient $(12,13,15,23)$. Since the research design that incorporates blinded examiners is a more powerful design, the lack of significant differences between the TMJ arthrocentesis group and the TMJ arthrocentesis with hyaluronic supplementation group may be a more accurate assessment of the benefit of HA. However, until a rigorous research design is implemented to test HA as a potential benefit after TMJ arthrocentesis for each sex, it remains unclear if this expensive supplement should be used for managing TMJ pain and mandibular mobility restriction.

\section{Platelet rich plasma supplementation after TMF arthrocentesis to reduce inflammation and stimulate $\mathrm{HA}$ synthesis and repair damaged tissue}

Only two RCT studies have been published using plateletrich plasma (PRP) as a supplement after TMJ arthrocentesis. PRP has been used in one study involving TMJ osteoarthritis patients (11). The benefit of PRP is the potential repair of damaged tissue within the joint by stimulating mesenchymal stem cell proliferation and promoting chondrocyte differentiation (40) and inducing new bone formation (41). Two groups of patients were studied where one group received TMJ arthrocentesis while the other group had the arthrocentesis procedure supplemented by PRP and then had weekly PRP injections for four more treatments. No statistically significant differences were found between the two groups for primary outcome measures of resting pain intensity or mandibular mobility improvement. However, a statistically significant improvement in masticatory efficiency (determined by VAS assessment) was found for the PRP group compared to no supplementation. The two groups were not the same sample size (12 vs. 18 subjects) and did not have the same proportion of females to males.

The other RCT studied PRP combined with calcium to release platelet-rich growth factors (also known as plasma-rich in growth factors or PRGF) and the effect of these growth factors was compared to no supplement after TMJ arthrocentesis (22). PRP growth factors stimulate the regeneration of new cartilage and bone (42) and stimulate the release of HA to lubricate the joint and reduce inflammation (43). The focus of this study was on patients with TMJ disk displacement without reduction and primary outcome measures were resting pain intensity and mandibular mobility. Statistically significant improvement was found for pain and maximum interincisal opening as well as masticatory efficiency. This study examined patients with only unilateral disk displacement without reduction and this inclusion criterion may have allowed the detection of differences between arthrocentesis groups without and with supplementation. However, experimental bias was not well controlled since a placebo was not included in the design and the examiner and the patient were not blinded to the patient's group assignment.

\section{Conclusions regarding the efficacy of supplements after TMF arthrocentesis}

Most of the RCTs that compared TMJ arthrocentesis with 
TMJ arthrocentesis with a supplement were low quality evidence since they were not well controlled, did not have adequate inclusion and exclusion criteria and did not adequately design the study to minimize experimental bias. The one high-quality evidence RCT that was designed to test subjects with confirmed unilateral TMJ intracapsular pain had a strong experimental design found a significant effect of a steroid supplement (triamcinolone acetonide). The steroid group had reduced chewing pain compared to the control group and the steroid normalized pain and mandibular mobility over TMJ arthrocentesis alone for the three month monitoring in female subjects. Further studies that include a group of males and a longer followup time using steroid supplementation would be valuable to determine the efficacy of this approach. However, it must be emphasized that steroid supplementation must be used sparingly since repeated joint exposure can cause bone resorption and remodeling.

HA as a post-TMJ arthrocentesis supplement has been the most intensely studied using RCTs. HA supplementation was not found to be significantly different from arthrocentesis alone in the moderate quality evidence RCT studies that included blinded examiners but these studies did not have sufficient inclusion and exclusion criteria to study a homogeneous sample. Most studies combined both sexes as a group to report outcomes and included unilateral and bilateral TMJ pain as inclusion criteria. At this time, the use of HA as supplement does not appear to provide additional efficacy over arthrocentesis alone.

PRP supplementation after TMJ arthrocentesis compared to arthrocentesis alone was examined in two RCT studies with one study finding significant differences between groups while the second study did not find differences. Studies using PRP or stimulated PRP to release growth factors are gaining more attention due to the multiple effects within the joint, particularly the potential to repair bone and cartilage from the growth factors released by platelets. The use of autologous sources to repair damaged tissue has the advantage of tissue compatibility and investigations into PRP supplementation after TMJ arthrocentesis appear promising but further study is necessary to ascertain the PRP efficacy.

\section{TMF arthrocentesis studies on methodology}

There have been multiple studies that have examined various modifications to the TMJ arthrocentesis technique to improve patient outcomes. For example, multiple studies have examined the theoretical basis for determining the necessary volume of the flushing medium to eliminate inflammatory mediators within the TMJ using TMJ computer modeling (35) or a protein recovery method (34). Both of the studies concluded that approximately 100-109 mL of flushing medium would be necessary to replace $100 \%$ of synovial fluid or remove proteins and proteases to below physiologic thresholds for proinflammatory cytokines. The joint modeling study also supported a two-needle lavage rather than a single needle-two cannula system to effectively lavage the superior joint space.

Needle placement using guided techniques such as ultrasound have been advocated over the "blind" method of needle placement due to increased visualization of the soft tissues. However, no studies have found that increased visualization is necessary for appropriate needle placement in the superior joint space and that outcome measures were no different using either technique (44-46).

\section{Conclusions}

Based on a review of RCTs, TMJ arthrocentesis without supplements is an effective technique to reduce resting pain intensity and chewing pain and increase mandibular mobility when usual treatment regimens such as pharmacotherapy, physical modalities, behavioral management and occlusal appliance therapy have not been successful. It was also concluded that additional imaging techniques such as ultrasound were not required to appropriately place the needles within the superior joint space to perform the procedure. Also, based on clinical monitoring of TMJ inflammatory mediators and TMJ modeling, the two-needle technique using at least $100 \mathrm{~mL}$ of saline or lactated Ringers had the most efficient outcome for lavaging the joint.

Intracapsular supplementation after arthrocentesis has been supported by a very limited number of moderate to high-quality evidence RCTs for a steroid (triamcinolone acetonide) and for PRP growth factors. HA supplementation has not been shown to be efficacious by moderate quality evidence RCTs although these trials had design deficiencies. After review of the available TMJ arthrocentesis RCTs, there is a need for additional RCTs that address the deficiencies that have been described in this review to adequately identify the most appropriate supplementation for a specific TMJ clinical condition and to examine supplementations that may be sex specific. Successfully addressing these issues may provide a more efficacious, evidence-based approach to the practicing 
clinician for management of intracapsular pain, disk mobility conditions and improved joint remodeling after osteoarthritis.

\section{Acknowledgments}

Funding: None.

\section{Footnote}

Provenance and Peer Review: This article was commissioned by the Guest Editors (Stephen Feinberg and Louis Mercuri) for the series "Temporomandibular Joint Disorders Diagnosis and Management - What Does the Future Hold?" published in Frontiers of Oral and Maxillofacial Medicine. The article has undergone external peer review.

Conflicts of Interest: Both authors have completed the ICMJE uniform disclosure form (available at https://fomm. amegroups.com/article/view/10.21037/fomm-20-51/coif). The series "Temporomandibular Joint Disorders Diagnosis and Management - What Does the Future Hold?" was commissioned by the editorial office without any funding or sponsorship. The authors have no other conflicts of interest to declare.

Ethical Statement: The authors are accountable for all aspects of the work in ensuring that questions related to the accuracy or integrity of any part of the work are appropriately investigated and resolved.

Open Access Statement: This is an Open Access article distributed in accordance with the Creative Commons Attribution-NonCommercial-NoDerivs 4.0 International License (CC BY-NC-ND 4.0), which permits the noncommercial replication and distribution of the article with the strict proviso that no changes or edits are made and the original work is properly cited (including links to both the formal publication through the relevant DOI and the license). See: https://creativecommons.org/licenses/by-nc-nd/4.0/.

\section{References}

1. Nitzan DW, Dolwick MF, Martinez GA.

Temporomandibular joint arthrocentesis: a simplified treatment for severe, limited mouth opening. J Oral Maxillofac Surg 1991;49:1163-7.

2. Alpaslan GH, Alpaslan C. Efficacy of temporomandibular joint arthrocentesis with and without injection of sodium hyaluronate in treatment of internal derangements. J Oral Maxillofac Surg 2001;59:613-8.

3. Aktas I, Yalcin S, Sencer S. Intra-articular injection of tenoxicam following temporomandibular joint arthrocentesis: a pilot study. Int J Oral Maxillofac Surg 2010;39:440-5.

4. Fridrich KL, Wise JM, Zeitler DL. Prospective comparison of arthroscopy and arthrocentesis for temporomandibular joint disorders. J Oral Maxillofac Surg 1996;54:816-20.

5. Goudot P, Jaquinet AR, Hugonnet S, et al. Improvement of pain and function after arthroscopy and arthrocentesis of the temporomandibular joint: a comparative study. J Craniomaxillofac Surg 2000;28:39-43.

6. Huddleston Slater JJ, Vos LM, Stroy LP, et al. Randomized trial on the effectiveness of dexamethasone in TMJ arthrocentesis. J Dent Res 2012;91:173-8.

7. Manfredini D, Rancitelli D, Ferronato G, et al. Arthrocentesis with or without additional drugs in temporomandibular joint inflammatory-degenerative disease: comparison of six treatment protocols* ${ }^{*} \mathrm{~J}$ Oral Rehabil 2012;39:245-51.

8. Tabrizi R, Karagah T, Arabion H, et al. Outcomes of arthrocentesis for the treatment of internal derangement pain: with or without corticosteroids? J Craniofac Surg 2014;25:e571-5.

9. Cömert Kiliç S. Does Injection of Corticosteroid After Arthrocentesis Improve Outcomes of Temporomandibular Joint Osteoarthritis? A Randomized Clinical Trial. J Oral Maxillofac Surg 2016;74:2151-8.

10. Comert Kilic S, Gungormus M. Is arthrocentesis plus platelet-rich plasma superior to arthrocentesis plus hyaluronic acid for the treatment of temporomandibular joint osteoarthritis: a randomized clinical trial. Int J Oral Maxillofac Surg 2016;45:1538-44.

11. Comert Kilic S, Gungormus M, Sumbullu MA. Is Arthrocentesis Plus Platelet-Rich Plasma Superior to Arthrocentesis Alone in the Treatment of Temporomandibular Joint Osteoarthritis? A Randomized Clinical Trial. J Oral Maxillofac Surg 2015;73:1473-83.

12. Patel P, Idrees F, Newaskar V, et al. Sodium hyaluronate: an effective adjunct in temporomandibular joint arthrocentesis. Oral Maxillofac Surg 2016;20:405-10.

13. Bouloux GF, Chou J, Krishnan D, et al. Is Hyaluronic Acid or Corticosteroid Superior to Lactated Ringer Solution in the Short-Term Reduction of Temporomandibular Joint Pain After Arthrocentesis? Part 1. J Oral Maxillofac Surg 
2017;75:52-62.

14. Gorrela H, Prameela J, Srinivas G, et al. Efficacy of Temporomandibular Joint Arthrocentesis with Sodium Hyaluronate in the Management of Temporomandibular Joint Disorders: A Prospective Randomized Control Trial. J Maxillofac Oral Surg 2017;16:479-84.

15. Ozdamar SM, Alev B, Yarat A. The impact of arthrocentesis with and without hyaluronic acid injection in the prognosis and synovial fluid myeloperoxidase levels of patients with painful symptomatic internal derangement of temporomandibular joint: a randomised controlled clinical trial. J Oral Rehabil 2017;44:73-80.

16. Tatli U, Benlidayi ME, Ekren O, et al. Comparison of the effectiveness of three different treatment methods for temporomandibular joint disc displacement without reduction. Int J Oral Maxillofac Surg 2017;46:603-9.

17. Bergstrand S, Ingstad HK, Moystad A, et al. Long-term effectiveness of arthrocentesis with and without hyaluronic acid injection for treatment of temporomandibular joint osteoarthritis. J Oral Sci 2019;61:82-8.

18. Öhrnell Malekzadeh B, Johansson Cahlin B, Widmark G. Conservative therapy versus arthrocentesis for the treatment of symptomatic disk displacement without reduction: a prospective randomized controlled study. Oral Surg Oral Med Oral Pathol Oral Radiol 2019;128:18-24.

19. Dolwick MF, Diaz D, Freburg-Hoffmeister DL, et al. A Randomized Double Blind Placebo Controlled Study of the Efficacy of Steroid Supplementation After Temporomandibular Joint Arthrocentesis. J Oral Maxillofac Surg 2020;78:1088-99.

20. Marzook HAM, Abdel Razek AA, Yousef EA, et al. Intra-articular injection of a mixture of hyaluronic acid and corticosteroid versus arthrocentesis in TMJ internal derangement. J Stomatol Oral Maxillofac Surg 2020;121:30-4.

21. Rao JKD, Sharma A, Kashyap R, et al. Comparison of efficacy of sodium hyaluronate and normal saline arthrocentesis in the management of internal derangement of temporomandibular joints - A prospective study. Natl J Maxillofac Surg 2019;10:217-22.

22. Toameh MH, Alkhouri I, Karman MA. Management of patients with disk displacement without reduction of the temporomandibular joint by arthrocentesis alone, plus hyaluronic acid or plus platelet-rich plasma. Dent Med Probl 2019;56:265-72.

23. Yapici-Yavuz G, Simsek-Kaya G, Ogul H. A comparison of the effects of Methylprednisolone Acetate, Sodium Hyaluronate and Tenoxicam in the treatment of non- reducing disc displacement of the temporomandibular joint. Med Oral Patol Oral Cir Bucal 2018;23:e351-8.

24. Hegab AF, Ali HE, Elmasry M, et al. PlateletRich Plasma Injection as an Effective Treatment for Temporomandibular Joint Osteoarthritis. J Oral Maxillofac Surg 2015;73:1706-13.

25. Abbasgholizadeh ZS, Evren B, Ozkan Y. Evaluation of the efficacy of different treatment modalities for painful temporomandibular disorders. Int J Oral Maxillofac Surg 2020;49:628-35.

26. Yilmaz O, Korkmaz YT, Tuzuner T. Comparison of treatment efficacy between hyaluronic acid and arthrocentesis plus hyaluronic acid in internal derangements of temporomandibular joint. J Craniomaxillofac Surg 2019;47:1720-7.

27. Sipahi A, Satilmis T, Basa S. Comparative study in patients with symptomatic internal derangements of the temporomandibular joint: analgesic outcomes of arthrocentesis with or without intra-articular morphine and tramadol. Br J Oral Maxillofac Surg 2015;53:316-20.

28. Singh RK, Pal US, Goyal P, et al. TMJ Arthrocentesis Alone and in Combination with Duloxetine in Temporomandibular Joint Pain. J Maxillofac Oral Surg 2018;17:270-5.

29. Hosgor H, Bas B, Celenk C. A comparison of the outcomes of four minimally invasive treatment methods for anterior disc displacement of the temporomandibular joint. Int J Oral Maxillofac Surg 2017;46:1403-10.

30. Bas B, Kazan D, Kutuk N, et al. The Effect of Exercise on Range of Movement and Pain After Temporomandibular Joint Arthrocentesis. J Oral Maxillofac Surg 2018;76:1181-6.

31. Sahlstrom LE, Ekberg EC, List T, et al. Lavage treatment of painful jaw movements at disc displacement without reduction. A randomized controlled trial in a short-term perspective. Int J Oral Maxillofac Surg 2013;42:356-63.

32. Baker Z, Eriksson L, Englesson Sahlstrom L, et al. Questionable effect of lavage for treatment of painful jaw movements at disc displacement without reduction: a 3 -year randomised controlled follow-up. J Oral Rehabil 2015;42:742-50.

33. Gurung T, Singh RK, Mohammad S, et al. Efficacy of arthrocentesis versus arthrocentesis with sodium hyaluronic acid in temporomandibular joint osteoarthritis: A comparison. Natl J Maxillofac Surg 2017;8:41-9.

34. Zardeneta G, Milam SB, Schmitz JP. Elution of proteins by continuous temporomandibular joint arthrocentesis. J Oral Maxillofac Surg 1997;55:709-16. 
35. Zhu P, Lin H, Zhou Q, et al. Dynamic Evaluation of Lavage Efficacy in Upper Compartment of the Temporomandibular Joint. J Oral Maxillofac Surg 2017;75:276-83.

36. Dworkin SF, LeResche L. Research diagnostic criteria for temporomandibular disorders: review, criteria, examinations and specifications, critique. J Craniomandib Disord 1992;6:301-55.

37. Dolwick MF, Widmer CG. Orthognathic Surgery as a Treatment for Temporomandibular Disorders. Oral Maxillofac Surg Clin North Am 2018;30:303-23.

38. Dworkin SF, Huggins KH, Le Resche L, et al. Epidemiology of signs and symptoms in temporomandibular disorders: clinical signs in cases and controls. J Am Dent Assoc 1990;120:273-81.

39. Plesh O, Curtis DA, Hall LJ, et al. Gender difference in jaw pain induced by clenching. J Oral Rehabil 1998;25:258-63.

40. Mishra A, Tummala P, King A, et al. Buffered platelet-rich plasma enhances mesenchymal stem cell proliferation and chondrogenic differentiation. Tissue Eng Part C Methods 2009; 15:431-5.

41. Han B, Woodell-May J, Ponticiello M, et al. The effect of thrombin activation of platelet-rich plasma on

doi: $10.21037 /$ fomm-20-51

Cite this article as: Dolwick MF, Widmer CG. Temporomandibular joint arthrocentesis: an evidence-based review. Front Oral Maxillofac Med 2020;2:26. demineralized bone matrix osteoinductivity. J Bone Joint Surg Am 2009;91:1459-70.

42. Fortier LA, Barker JU, Strauss EJ, et al. The role of growth factors in cartilage repair. Clin Orthop Relat Res 2011;469:2706-15.

43. Anitua E, Sanchez M, Nurden AT, et al. Platelet-released growth factors enhance the secretion of hyaluronic acid and induce hepatocyte growth factor production by synovial fibroblasts from arthritic patients. Rheumatology (Oxford) 2007;46:1769-72.

44. Sivri MB, Ozkan Y, Pekiner FN, et al. Comparison of ultrasound-guided and conventional arthrocentesis of the temporomandibular joint. Br J Oral Maxillofac Surg 2016;54:677-81.

45. Antony PG, Sebastian A, D A, et al. Comparison of clinical outcomes of treatment of dysfunction of the temporomandibular joint between conventional and ultrasound-guided arthrocentesis. Br J Oral Maxillofac Surg 2019;57:62-6.

46. Bhargava D, Thomas S, Pawar P, et al. Ultrasound-guided arthrocentesis using single-puncture, double-lumen, single-barrel needle for patients with temporomandibular joint acute closed lock internal derangement. Oral Maxillofac Surg 2019;23:159-65. 